\title{
Rheumatoid arthritis and pregnancy; not only for rheumatologists interested in female health issues
}

\section{Radboud J E M Dolhain}

In this issue of the Annals of the Rheumatic Diseases Wallenius et al ${ }^{1}$ report an increase in the onset of rheumatoid arthritis (RA) and other arthritides just after delivery (see article on page 332). They have shown this by linking two national registers-namely, the Norwegian Disease-Modifying Antirheumatic Drug (NOR-DMARD) Register and the Medical Birth Register of Norway. This report confirms previous studies ${ }^{2-4}$ on RA and additionally extends our knowledge to other forms of arthritis. Owing to the low incidence of RA occurring simultaneously or just after pregnancy, the previous studies on this subject were mainly of a matched case-control design. ${ }^{2}{ }^{4}$ Since every study design carries the risk of introducing its own type of bias, conformation of this finding in a different setting is important.

In these previous studies it has been shown that the increased incidence of RA after delivery is accompanied by a lower incidence during pregnancy. ${ }^{2}$ This suggests that the increased incidence of RA after delivery is not a true increased incidence, but rather that the occurrence of RA is postponed until after delivery.

How can these data be interpreted? It is clear that they can be seen in the scope of the well-known ameliorating effect of pregnancy on the course of existing RA and the flare-up of disease activity in the postpartum period. Since the first description of this phenomenon by Hench in $1938,{ }^{5}$ this finding has been reconfirmed in several studies, ${ }^{67}$ most recently in a large prospective study from before conception and onwards. ${ }^{8}$ In this first large prospective study using validated instruments to determine disease activity, ${ }^{9}$ it was shown that almost half of women responded during pregnancy and more than one-third flared after delivery despite

Correspondence to: Dr R J E M Dolhain, Department of Rheumatology, Office 961a, Erasmus MC, University Medical Centre Rotterdam, PO Box 2040, 3000 CA

Rotterdam, The Netherlands; R.Dolhain@erasmusmc.nl the fact that DMARDs were again started. Several theories have been proposed to explain this beneficial effect of pregnancy on RA disease activity, including, for example, an increase in regulatory T cells, ${ }^{10}$ a shift from Th1 to Th2 cells ${ }^{11}$ and changes in glycosylation of $\operatorname{IgG} .{ }^{12}$ However, owing to the difficulty in performing a prospective study in pregnant RA women, the majority of these studies are small and hence do not allow a comparison of patients with RA who improve during pregnancy with those who do not. Therefore, it can often not be concluded from these studies whether the observed changes only represent a general adaptation of the immune system during pregnancy or whether these changes are also responsible for the ameliorating effect of pregnancy on RA.

The observed adaptations of the immune system during pregnancy are, in general, thought to result from hormonal changes associated with pregnancy. In this respect the effect of oestrogens, progesterone and cortisol have been most widely studied. Briefly, in high concentrations, compatible with pregnancy, oestrogens inhibit T-cell function; in particular, Th1 cells, inhibit the production of interleukin (IL)1, IL6, IL12 and tumour necrosis factor by monocytes/macrophages and dendritic cells, but stimulate (auto)antibody production. Progesterone inhibits production of IL1, IL6 and IL12 and stimulates Th2 effects, whereas cortisol is thought to have a general immunosuppressive effect. ${ }^{13}$

That pregnancy hormones exert both immunostimulatory and immunosuppressive properties has often been used as an explanation for the different impact that pregnancy may have on various autoimmune diseases, depending on the pathogenic mechanisms involved. Despite all the conflicting results there is some agreement that the frequency of flares is higher in pregnant than in non-pregnant patients with systemic lupus erythematosus. ${ }^{1415}$ The role for oestrogens in the pregnancy related flares in systemic lupus erythematosus has, however, still not been fully established. ${ }^{13}{ }^{16}$ Also, the recent observation that patients with RA who are positive for anti-citrullinated protein antibody (ACPA) and rheumatoid factor are less likely to improve during pregnancy indicates that the beneficial effect of pregnancy depends upon the disease mechanism involved. ${ }^{17}$

In line with the hypothesis that changes of the immune system are responsible for the beneficial effect of pregnancy on RA, the flare of RA after delivery is often explained by a gradual returning of the immune system to a prepregnancy state after delivery. Owing to a lack of studies, it is not known whether such a gradual returning of the immune system to this pre-pregnancy state occurs. On the contrary, it might very well be that like pregnancy also the postpartum period represents a specific state of the immune system that differs from the state before pregnancy. In this respect, a role for high prolactin levels, associated with breastfeeding, has been suggested. Prolactin is thought to be involved in the breakdown of B-cell tolerance, enhancing cell proliferation and the development of antigen presenting cells. It promotes immunoglobulin production and the production of proinflammatory cytokines. ${ }^{18}$ In RA, breastfeeding has been associated with the occurrence of the postpartum flare. ${ }^{19}$ The interpretation of this association, however, is difficult, since women who breastfeed are in general not receiving medication and hence are more likely to flare.

We have recently found that the postpartum period has a specific influence on the levels of mannose binding lectin $(\mathrm{MBL}) . \mathrm{MBL}$ is a complement factor that initiates the lectin pathway of complement. During pregnancy MBL levels are increased, but after delivery MBL levels decline to such an extent that some women even become MBL deficient, before returning to normal in the next few months. ${ }^{20}$ Although a role for MBL in the pathogenesis of RA has been questioned, ${ }^{21}$ this finding clearly illustrates that the changes of the immune system in the postpartum period do not simply reflect a gradual returning of the immune system to its pre-pregnancy homoeostasis, but may rather reflect a specific situation. Such a specific situation could, from an immunological point of view, be an explanation for the increased incidence of RA and other forms of chronic arthritides in the postpartum period. However, 
more epidemiological data are needed to determine whether this is a true increased incidence or whether rather the incidence of RA and other forms of arthritis is postponed to after delivery. Unfortunately, this question is not answered in the article of Wallenius et al. ${ }^{1}$

What can we learn from studies on pregnancy and RA? Our concept of the syndrome that we call RA has changed dramatically during the past decade. One of the most revolutionary concepts is that RA is not a fixed entity that just occurs, but has a long preclinical period. ${ }^{22}$ This is supported by the finding that RA-specific antibodies like ACPA and rheumatoid factor can be found years before the actual onset of RA. ${ }^{23}$ Although these autoantibodies are thought to be important in the pathogenesis of RA, their actual presence does not seem to be sufficient to result in arthritis. ${ }^{24}$

All these findings have led to a new pathogenic model for RA. According to this model RA results from a complex gene-environment interaction, in which RA only develops after the immune systems has been triggered by several environmental factors, a process which may take years. ${ }^{22}$ One of the environmental factors that has been clearly shown to trigger RA is smoking. ${ }^{25}$ Besides predisposing environmental factors, protecting environmental factors have also been described, including pregnancy. Although not reconfirmed in all studies, ${ }^{26}{ }^{27}$ women who have been pregnant have an approximately $50 \%$ reduced lifetime risk of developing RA compared with nulliparous women. ${ }^{28} 29$

If we now, in line with the aforementioned pathogenic model, consider RA as a long process from the preclinical period, to the occurrence of arthralgias and arthritis and, finally, the development of a symmetrical destructive polyarthritis, the effect of pregnancy on RA might be threefold. In the early stages it might reverse the disease process, later on it might postpone the onset of clinical symptoms and in the final stages it results in relief of clinical signs. The immunological mechanisms responsible, however, may be identical.
Viewed in such a way, the impact of studies like that of Wallenius et $a l^{1}$ goes beyond the field of rheumatologists interested in female health issues, but these studies may contribute to a better understanding of the fundamental question why one person gets RA and another does not. The Norwegian situation in which two national health registers are linked, offers unique opportunities for more research in this particular field and more interesting data can hence be expected.

\section{Competing interests: None.}

Provenance and peer review: Commissioned; externally peer reviewed.

Accepted 30 November 2009

Ann Rheum Dis 2010;69:317-318.

doi:10.1136/ard.2009.120741

\section{REFERENCES}

1. Wallenius $\mathbf{M}$, Skomsvoll JF, Irgens LM, et al. Postpartum onset of rheumatoid arthritis and other chronic arthritides: results from a patient register linked to a medical birth registry. Ann Rheum Dis 2010;69:332-6.

2. Lansink M, de Boer A, Dijkmans BA, et al. The onset of rheumatoid arthritis in relation to pregnancy and childbirth. Clin Exp Rheumatol 1993;11:171-4.

3. Oka M. Effect of pregnancy on the onset and course of rheumatoid arthritis. Ann Rheum Dis 1953;12:227-9.

4. Silman A, Kay A, Brennan P. Timing of pregnancy in relation to the onset of rheumatoid arthritis. Arthritis Rheum 1992;35:152-5.

5. Hench PS. The ameliorating effect of pregnancy on chronic atrophic (infectious rheumatoid) arthritis, fibrosis, and intermittent hydrarthrosis. Mayo Clin Proc 1938;13:161-7.

6. Barrett JH, Brennan P, Fiddler M, et al. Does rheumatoid arthritis remit during pregnancy and relapse postpartum? Results from a nationwide study in the United Kingdom performed prospectively from late pregnancy. Arthritis Rheum 1999;42:1219-27.

7. Nelson JL, Ostensen M. Pregnancy and rheumatoid arthritis. Rheum Dis Clin North Am 1997:23:195-212

8. de Man YA, Dolhain RJ, van de Geijn FE, et al. Disease activity of rheumatoid arthritis during pregnancy: results from a nationwide prospective study. Arthritis Rheum 2008:59:1241-8.

9. de Man YA, Hazes JM, van de Geijn FE, et al. Measuring disease activity and functionality during pregnancy in patients with rheumatoid arthritis. Arthritis Rheum 2007:57:716-22.

10. Forger F, Marcoli N, Gadola S, et al. Pregnancy induces numerical and functional changes of CD4+CD25 high regulatory T cells in patients with rheumatoid arthritis. Ann Rheum Dis 2008;67:984-90

11. Munoz-Valle JF, Vazquez-Del Mercado M, GarciaIglesias $T$, et al. $T(H) 1 / T(H) 2$ cytokine profile, metalloprotease- 9 activity and hormonal status in pregnant rheumatoid arthritis and systemic lupus erythematosus patients. Clin Exp Immunol 2003; 131:377-84.

12. Alavi A, Arden N, Spector TD, et al. Immunoglobulin $\mathrm{G}$ glycosylation and clinical outcome in rheumatoid arthritis during pregnancy. J Rheumatol 2000;27:1379-85

13. Straub RH. The complex role of estrogens in inflammation. Endocr Rev 2007;28:521-74.

14. Khamashta MA, Ruiz-Irastorza G, Hughes GR. Systemic lupus erythematosus flares during pregnancy. Rheum Dis Clin North Am 1997;23:15-30.

15. Petri M. Hopkins Lupus Pregnancy Center: 1987 to 1996. Rheum Dis Clin North Am 1997;23:1-13.

16. Petri M. Sex hormones and systemic lupus erythematosus. Lupus 2008;17:412-15

17. de Man YA, Bakker-Jonges LE, Dufour-van den Goorbergh DB, et al. Women with rheumatoid arthritis negative for anti-CCP and rheumatoid factor are more likely to improve during pregnancy, whereas in autoantibody-positive women autoantibody levels are not influenced by pregnancy. Ann Rheum Dis 2010;69:420-3.

18. Orbach H, Shoenfeld Y. Hyperprolactinemia and autoimmune diseases. Autoimmun Rev 2007:6:537-42

19. Barrett JH, Brennan P, Fiddler M, et al. Breastfeeding and postpartum relapse in women with rheumatoid and inflammatory arthritis. Arthritis Rheum 2000;43:1010-15

20. van de Geijn FE, Roos A, de Man YA, et al. Mannose-binding lectin levels during pregnancy: a longitudinal study. Hum Reprod 2007;22:362-71.

21. van de Geijn FE, Hazes JM, Geleijns K, et al. Mannose-binding lectin polymorphisms are not associated with rheumatoid arthritis - confirmation in two large cohorts. Rheumatology (Oxford) 2008:47:1168-71.

22. Klareskog L, Padyukov L, Ronnelid J, et al. Genes, environment and immunity in the development of rheumatoid arthritis. Curr Opin Immunol 2006;18:650-5.

23. Nielen MM, van Schaardenburg D, Reesink HW, et al. Specific autoantibodies precede the symptoms of rheumatoid arthritis: a study of serial measurements in blood donors. Arthritis Rheum 2004;50:380-6.

24. Cantaert T, De Rycke L, Bongartz T, et al. Citrullinated proteins in rheumatoid arthritis: crucial... but not sufficient! Arthritis Rheum 2006;54:3381-9.

25. Lundstrom E, Kallberg H, Alfredsson L, et al. Geneenvironment interaction between the DRB1 shared epitope and smoking in the risk of anti-citrullinated protein antibody-positive rheumatoid arthritis: all alleles are important. Arthritis Rheum 2009;60:1597-603.

26. Brun JG, Nilssen S, Kvale G. Breast feeding, other reproductive factors and rheumatoid arthritis. $\mathrm{A}$ prospective study. Br J Rheumatol 1995;34:542-6.

27. Hernandez Avila $\mathbf{M}$, Liang $\mathbf{M H}$, Willett $\mathbf{W C}$, et al. Reproductive factors, smoking, and the risk for rheumatoid arthritis. Epidemiology 1990;1:285-91.

28. Hazes JM, Dijkmans BA, Vandenbroucke JP, et al. Pregnancy and the risk of developing rheumatoid arthritis. Arthritis Rheum 1990;33:1770-5.

29. Spector TD, Roman E, Silman AJ. The pill, parity, and rheumatoid arthritis. Arthritis Rheum 1990;33:782-9. 